\title{
Análise da produção bibliográfica sobre atenção primária à saúde no Brasil em quatro periódicos selecionados
}

\section{| 1 Tatiana Wargas de Faria Baptista, \\ ${ }^{2}$ Márcia Cristina Rodrigues Fausto, ${ }^{3}$ Marcela Silva da Cunha |}

Resumo: O artigo analisa a produção bibliográfica em atenção primária no Brasil publicada em quatro revistas de grande circulação no campo da Saúde Coletiva - Cadernos de Saúde Pública, Revista de Saúde Pública, Ciência e Saúde Coletiva e Saúde em Debate -, com o intuito de identificar os conceitos utilizados, as abordagens dos estudos e os principais temas associados à discussão da atenção primária. A análise foi feita a partir da compreensão do contexto político-institucional da saúde, buscando correlações do momento da política com a produção de conhecimento. A análise abrangeu a discussão realizada nesses periódicos de 1977 até 2006. Verificou-se uma carência de estudos de abordagem conceitual e uma abundância de estudos de caso sem a promoção do diálogo entre autores e instituições, fragilizando essa área como produtora de conhecimento. Com este estudo, pretendese contribuir para o aprofundamento do debate teórico sobre o modelo assistencial com foco na atenção primária e identificar linhas de força das propostas, contribuindo para a consolidação do campo.

> Palavras-chave: atenção primária à saúde; atenção básica; saúde da família; produção de conhecimento.

\author{
1 Pesquisadora do Departamento \\ de Administração e \\ Planejamento e Saúde da Escola \\ Nacional de Saúde Pública \\ Sérgio Arouca da Fundação \\ Oswaldo Cruz (DAPS/ENSPI \\ FIOCRUZ). Endereço eletrônico: \\ twargas@ensp.fiocruz.br \\ 2 Pesquisadora da Escola de \\ Governo da Escola Nacional de \\ Saúde Pública Sérgio Arouca da \\ Fundação Oswaldo Cruz (EGS/ \\ ENSP/FIOCRUZ). \\ ${ }^{3}$ Mestranda em Saúde Pública \\ da Escola Nacional de Saúde \\ Pública Sérgio Arouca da \\ Fundação Oswaldo Cruz (DAPS/ \\ ENSP/FIOCRUZ)
}

Recebido em: 06/03/2008. Aprovado em: 28/07/2008. 
A atenção primária à saúde (APS) vem exercendo papel fundamental na organização das ações dos sistemas de saúde em diversos países desde meados do século XX, configurando-se de forma singular em cada realidade. A concepção e as práticas de saúde relativas à APS se alteraram ao longo do tempo, assim como são diferentes as percepções quanto a seu escopo para distintos atores envolvidos no campo da saúde.

Considerando a diversidade de sistemas de serviços de saúde existentes, Vuori (1986) apresenta pelo menos quatro diferentes formas de compreensão sobre a APS: como um conjunto de atividades; um nível de assistência; uma estratégia de organização do sistema de serviços; e como um princípio que deve nortear todas as ações desenvolvidas em um sistema de saúde.

Starfield (2002) considera que as várias formas de compreensão da APS apresentadas por Vuori não são excludentes e podem coexistir num mesmo sistema de saúde, não sendo possível identificar uma única definição que exprima o significado da APS. Para a autora, a APS deve ser compreendida como uma abordagem que forma a base e determina o trabalho de todos os outros níveis do sistema de saúde, organizando e racionalizando o uso de todos os recursos, tanto básicos como especializados, direcionados para a promoção, manutenção e melhora da saúde.

No caso brasileiro, a palavra primário(a), na língua portuguesa, traz em si diferentes possibilidades explicativas - o primeiro em uma lista ordenada; principal, central, essencial; primitivo, básico, sem refinamento. Os efeitos do problema terminológico sobre a compreensão da APS acarretam pelo menos três sentidos: pode evocar integração e interdependência do cuidado, pois embora seja principal ou essencial dentro de um sistema, subentende-se a existência e importância da sua relação com os demais níveis do cuidado; pode representar também um mínimo de atividades essenciais que os sistemas de saúde possam prover, tendo um sentido seletivo que exclui outros elementos cruciais do cuidado; pode ser interpretada como porta de entrada, o primeiro contato dos indivíduos com o sistema de saúde, exercendo assim a função de triagem para inclusão ou barreira às ações de maior complexidade tecnológica. Esta ambiguidade terminológica tem causado confusão, falta de clareza e ausência de consenso em relação ao sentido ou propósito da APS (FAUSTO, 2005). 
Este artigo surge no contexto deste debate com o intuito de aprofundar a discussão sobre as diferentes concepções e abordagens de atenção primária no Brasil. Ressalte-se que, no Brasil, o termo utilizado, tanto na política oficial como no âmbito acadêmico, tem sido "atenção básica", configurando-se numa estratégia de reorientação do modelo assistencial definida a partir de 1996. No artigo utilizaremos os termos atenção básica e atenção primária como sinônimos, entendendo que o primeiro expressa a concepção da política de saúde brasileira para o que internacionalmente se convencionou chamar de APS, o que exploraremos mais adiante.

Em estudo de revisão bibliográfica sobre APS na América Latina (PAHO, 2002) observou-se que há um número reduzido de estudos sobre o tema na região, especialmente no que se refere à noção de APS abrangente e integrada ao sistema de saúde, sugerindo, ainda que indiretamente, o predomínio da APS numa perspectiva seletiva e focalizadora. O relatório OPAS (PAHO, 2002) aponta que, em termos quantitativos, a produção bibliográfica sobre o tema APS realizada no Brasil se destaca e tem influenciado a produção nos demais países da América Latina. O documento técnico (CONILL; FAUSTO, 2007) sobre APS produzido pelo Intercâmbio EuroSocial Salud enfatiza a importância dos estudos que tratam da problemática da integração da APS, uma vez que a experiência latino-americana se mostra débil porque historicamente tem-se constituído como uma ação seletiva pouco articulada com outras ações do sistema de saúde. Isto reflete uma tendência dos sistemas na região. Discutir os problemas e perspectivas relacionados à condução dos modelos de atenção pautados na APS é crucial para os rumos desta proposta.

Relatório divulgado pela OPAS e OMS (PAHO, 2007) reforça a importância da disseminação da informação científica para a identificação dos mais efetivos caminhos para organizar, financiarl e ofertar cuidados em saúde adequados e equitativos. A análise sobre a tendência da produção científica na América Latina e Caribe no período de 2000 a 2005 disponível em diferentes bases de dados aponta que o Brasil tem importante participação na produção disseminada entre os países da região. Cerca de $50 \%$ da produção difundida nas bases Lilacs, Medline e SciELO são originárias do Brasil. Dos jornais indexados da América Latina e Caribe disponíveis nessas bases, $40 \%$ são publicações brasileiras.

Admitindo-se que a produção de conhecimento sobre APS realizada no Brasil tem influenciado a informação científica nos países da América Latina, torna-se 
relevante reconhecer quais são os marcos teóricos que caracterizam esta produção, assim como as lacunas de conhecimento que se apresentam e as perspectivas futuras para a produção nessa área temática.

Este artigo apresenta os resultados da análise da produção bibliográfica em atenção primária no Brasil publicada em quatro revistas de grande circulação no campo da Saúde Coletiva, com o intuito de identificar os principais elementos de discussão sobre a temática: conceitos utilizados, abordagens dos estudos e principais temas associados ao debate da APS. Pretende-se com este estudo contribuir para o aprofundamento do debate teórico sobre o modelo assistencial com o foco na APS e possibilitar o conhecimento do que existe de produção nessa área, identificando linhas de força das propostas e contribuindo para a consolidação do campo.

O texto está dividido em três partes. A primeira trata do marco teórico que localiza a discussão sobre APS na trajetória da política de saúde no Brasil. A segunda apresenta a metodologia do estudo, como e por que analisamos a produção bibliográfica de quatro periódicos. E a última discute os resultados da análise empreendida.

\section{Atenção primária: conceitos, trajetória e a experiência brasileira}

Em termos conceituais, foi a partir da I Conferência Internacional de Cuidados Primários em Saúde, realizada em 1978 em Alma-Ata, Cazaquistão, que se buscou uma definição de APS (FAUSTO, 2005). Assim, a Organização Mundial da Saúde (OMS) passou a compreender e divulgar os cuidados primários em saúde como:

Cuidados essenciais baseados em métodos práticos, cientificamente bem fundamentados e socialmente aceitáveis e em tecnologia de acesso universal para indivíduos e suas famílias na comunidade [...]. Além de serem o primeiro nível de contato de indivíduos, da família e da comunidade com o sistema nacional de saúde, aproximando ao máximo possível os serviços de saúde nos lugares onde o povo vive e trabalha, constituem também o primeiro elemento de um contínuo processo de atendimento em saúde. (OMS/UNICEF, 1979, p. 2).

Este conceito foi amplamente difundido por inúmeros trabalhos produzidos sobre APS, assim como influenciou as propostas de organização da atenção em diferentes sistemas de saúde.

Um ano após a Conferência de Alma-Ata, a Fundação Rockefeller financiou a realização da Conferência de Bellagio, a partir da qual se disseminou 
internacionalmente uma noção seletiva da APS, baseada na formulação de programas com objetivos focados em problemas específicos de saúde para atingir grupos populacionais em situação de pobreza (CUETO, 2003; MAGNUSSEN; JOLLY, 2004). A proposta surgiu da crítica feita à concepção de APS presente na Declaração de Alma-Ata, interpretada como uma concepção idealizada, muito ampla e com poucas chances de aplicabilidade.

A tensão discursiva entre essas duas vertentes percorreu toda a década de 1980 com reflexos na discussão sobre a organização dos serviços de saúde na década seguinte. A disseminação de programas seletivos de APS em países em desenvolvimento foi divulgada e financiada por agências e fundações internacionais. O principal objetivo era a extensão de cobertura a partir da oferta de ações de saúde simples e de baixo custo, principalmente em áreas rurais onde a população não tinha acesso ao sistema de saúde existente.

Nos anos 1990, o Banco Mundial assumiu papel central no debate internacional, com a publicação do relatório Investindo em Saúde, ofertando ideias que tinham como argumento a consolidação de sistemas de saúde capazes de responder a um conjunto mínimo de ações essenciais e a crítica à universalidade do acesso à saúde (BANCO MUNDIAL, 1993). No ano 2000, a OMS divulgou o relatório Sistemas de Saúde: melhorando a performance, com a defesa de políticas de saúde compatíveis com a capacidade de sustentação de cada Estado, prezando-se pela qualidade da atenção à população coberta pelo sistema (WHO, 2004). Um novo documento é apresentado pela OMS em 2003 (OMS, 2003) visando à retomada dos princípios da APS formulados na Conferência de Alma-Ata e com o revigoramento da concepção de cuidado integrado.

No Brasil, as primeiras experiências em APS são anteriores à Conferência de Alma-Ata e se desenvolveram nos anos 1960, e mais amplamente nos anos 1970, a partir dos programas de integração docente-assistencial dos Departamentos de Medicina Preventiva, vinculados às escolas de medicina (FAUSTO, 2005). Em meados dos anos 1970, as experiências começaram a ganhar maior expressão, influenciando na formulação de novas abordagens e formas de organização da atenção no país (FAUSTO, 2005).

$\mathrm{Na}$ década de 1980, enquanto muitos países da América Latina e outros países em desenvolvimento implementavam programas focalizados de APS, conforme recomendação de agências internacionais, o Brasil avançou no sentido da reforma sanitária sob outros princípios, tais como: o da universalização, da equidade no 
acesso e da integralidade das ações dos serviços de saúde, refletindo o contexto de redemocratização do Estado. Surgem experiências inovadoras nos sistemas locais de saúde e algumas propostas ganharam visibilidade nacional, tornando-se exemplos de modelos bem-sucedidos (Cidade Saudável, Defesa da Vida, SILOS, Distritos Sanitários, Vigilância à Saúde), influenciando a organização da atenção à saúde nos diversos municípios e regiōes brasileiras.

Na segunda metade da década de 1990, com a definição da Norma Operacional Básica do Ministério da Saúde de 1996 (NOB 01/96), o Governo Federal passou a incentivar financeiramente a implantação do Programa Saúde da Família (PSF) e do Programa Agentes Comunitários de Saúde (PACS), formulados desde o início da década em alguns municípios como estratégia para reorientação do modelo na rede pública de saúde.

Com a NOB 96, o debate sobre "atenção primária" ressurgiu e ganhou expressão na discussão política e acadêmica no Brasil. Neste sentido, podemos dizer que o PACS e o PSF foram as janelas de oportunidade (KINGDOM, 1995) para a ascensão e retomada da APS na agenda da política nacional de saúde, retratando um primeiro momento de inflexão da política de atenção básica na política federal da saúde nos anos 90 (LEVCOVITZ; GARRIDO, 1996; SOUZA, 2002). Um segundo momento de inflexão se configurou a partir de 1998, quando a NOB 96 foi implementada e se iniciou um processo de expansão maciça do PSF no território nacional. Um terceiro momento de inflexão configurou-se a partir do ano de 2003, com a implantação do Projeto de Expansão e Consolidação da Estratégia de Saúde da Família para os grandes centros urbanos (PROESF).

Com a expansão do PSF, surgem diagnósticos sobre os desafios e impasses na construção de um modelo assistencial pautado no PSF e o debate político e acadêmico indica a necessidade de fortalecimento da atenção básica numa perspectiva que não se restrinja ao modelo do PSF, numa tentativa de revalorização da concepção de APS, buscando o diálogo com o debate internacional (FAUSTO, 2005; GIL, 2006; CANESQUI; OLIVEIRA, 2002). Nesse contexto, renova-se a discussão sobre APS. Um conceito bastante trabalhado no âmbito internacional desde a década de 70, mas que foi utilizado com menor expressão no processo da reforma sanitária no Brasil, que tinha como principal foco a garantia do direito universal à saúde e a rejeição à concepção de uma atenção primária seletiva. 
No percurso histórico da política de saúde nacional instiga-nos, portanto, entender a compreensão sobre APS predominante na literatura brasileira na área da Saúde Coletiva.

\section{Estratégias metodológicas}

Este estudo parte do levantamento da produção bibliográfica sobre atenção primária e saúde da família, realizado no contexto da pesquisa de Linha de Base do PROESF. O levantamento reuniu artigos em periódicos nacionais, disponíveis na base de dados da BIREME (Biblioteca Virtual em Saúde) até dezembro de 2005. A busca foi feita a partir das seguintes palavras-chave e descritores: atenção primária, atenção primária à saúde, assistência primária, cuidados primários de saúde, atenção básica, saúde da família, Programa Saúde da Família, agentes comunitários de saúde e avaliação da atenção básica. Após a coleta das referências, foi feita a verificação das duplicidades e excluídos os estudos, que apesar de remetidos à atenção primária, tinham como foco estritamente a abordagem clínica e o levantamento epidemiológico neste âmbito.

A revisão bibliográfica totalizou 230 referências com 98 artigos (43\%) publicados em quatro periódicos - Cadernos de Saúde Pública (CSP/FIOCRUZ), Revista de Saúde Pública (RSP/USP), Ciência e Saúde Coletiva (CSC/ABRASCO), Revista Saúde em Debate (SD/CEBES) -, e os demais em periódicos diversos. A partir da constatação do quantitativo expressivo de artigos veiculados nos quatro periódicos identificados e da importância dos mesmos no campo da Saúde Coletiva, definiuse como proposta de estudo a análise qualitativa dos artigos produzidos no âmbito destas revistas, tendo como objetivo central identificar os principais elementos de discussão sobre a temática da APS no Brasil, ressaltando os conceitos utilizados, as abordagens dos estudos e os principais temas associados ao debate da APS.

Numa segunda etapa do trabalho, foi feita a revisão de todos os volumes editados pelas revistas com o objetivo de identificar os estudos que não foram captados na busca no ambiente virtual, especialmente aqueles artigos das décadas de 1960, 1970 e início da década de 1980 que não constavam de palavras-chave. Os artigos identificados foram incluídos mediante a leitura do resumo. Com a revisão, foi feita também a inclusão dos artigos publicados no ano de 2006. Ao final somaram-se mais 31 artigos, totalizando 129 artigos para análise (tabela 1). 
Tabela 1 - Produção bibliográfica em APS em quatro periódicos nacionais por ano da publicação

\begin{tabular}{|c|c|c|c|c|c|}
\hline ANO/ VEICULO & $\mathrm{CSC}$ & CSP & RSP & SD & Total \\
\hline 1977 & & & 1 & & 1 \\
\hline 1984 & & & 1 & & 1 \\
\hline 1985 & & & 1 & & 1 \\
\hline 1987 & & & 1 & & 1 \\
\hline 1989 & & 2 & & & 2 \\
\hline 1991 & & & 1 & & 1 \\
\hline 1992 & & 1 & & & 1 \\
\hline 1994 & & & 1 & 1 & 2 \\
\hline 1996 & & & & 1 & 1 \\
\hline 1997 & & 2 & 1 & & 3 \\
\hline 1998 & & 4 & & & 4 \\
\hline 1999 & 1 & & 1 & & 2 \\
\hline 2000 & & 2 & 3 & & 5 \\
\hline 2001 & 1 & 1 & 2 & 1 & 5 \\
\hline 2002 & 5 & 6 & 2 & 5 & 18 \\
\hline 2003 & 4 & 6 & 1 & 4 & 15 \\
\hline 2004 & 4 & 10 & & 2 & 16 \\
\hline 2005 & 10 & 7 & 6 & & 23 \\
\hline 2006 & 13 & 13 & 1 & & 27 \\
\hline Total & 38 & 54 & 23 & 14 & 129 \\
\hline
\end{tabular}

Fonte: Base de dados da produção dos periódicos Revista de Saúde Pública/USP, Cadernos de Saúde Pública/FIOCRUZ, Ciência e Saúde Coletiva/ABRASCO e Revista Saúde em Debate/CEBES. 
Cabe ressaltar que cada periódico tem início temporal próprio. A RSP inicia-se no ano de 1967, os CSP têm seu primeiro número editado no ano de 1973, a RSD no ano de 1976 e a CSC no ano de 1996. Identificaram-se, nestes periódicos, o primeiro artigo nesta temática no ano de 1977 e um aumento expressivo de referências a partir da década de 90, o que reflete, por um lado, a tendência da produção, e, por outro, a trajetória da política de saúde. A correlação entre produção acadêmica e política de saúde foi um elemento de análise ressaltado no contexto deste estudo.

Todos os artigos selecionados foram analisados a partir das seguintes categorias: a) contexto do artigo - ano da publicação e momento da política; b) temática - foco principal dos estudos; c) abrangência - definição e atributos da APS; d) autoria - autores e instituições; e) abordagem do estudo - internacional, nacional, local, teórico-conceitual.

\section{A produção em APS no Brasil}

A produção bibliográfica em APS aumentou de forma relevante a partir dos anos 2000 nos quatro periódicos analisados (tabela 1), tendo como contexto o incentivo para a reorganização do modelo assistencial pautado na atenção básica e na expansão do PSF.

Nos anos 70 e 80, a produção nacional incorporou a orientação para a extensão da cobertura, mas também a preocupação com a afirmação da garantia da assistência primária na lógica do cuidado integral em saúde e da medicina integral, com a negação de qualquer possibilidade de configuração de uma política seletiva ou excludente. A discussão de extensão da cobertura aparece atrelada ao debate de reconfiguração do modelo assistencial na perspectiva da garantia do direito à saúde (NORONHA et al., 1977; NOGUEIRA, 1984).

Ao longo da década de 1980, os artigos tenderam a enfatizar os estudos sobre a avaliação da assistência com o foco na organização do sistema de serviços de saúde (quadro 1) - tendência que se manteve na produção durante os anos $90 \mathrm{e}$ que de alguma forma foi se alterando à medida que as discussões sobre modelos de atenção ganharam expressão na condução da política nacional de saúde. Temas como modelo assistencial, medicina familiar e comunitária, educação popular e participação comunitária começam a ser explorados, revisitando aspectos relacionados à construção da política na ponta do sistema. 

em quatro periódicos nacionais por ano da publicação

\begin{tabular}{|c|c|c|c|}
\hline Anos & Principais temas & Sub-temas & $\begin{array}{c}\mathrm{N}^{\circ} \text { de } \\
\text { artigos }\end{array}$ \\
\hline 1977 & Assistência à saúde & Medicina Integral & 1 \\
\hline 1984 & Assistência à saúde & Saúde integral do trabalhador & 1 \\
\hline 1985 & Avaliação da assistência & Padrões de morbidade & 1 \\
\hline 1987 & Assistência à saúde & Saúde mental & 1 \\
\hline \multirow[t]{2}{*}{1989} & Assistência à saúde & Agentes de saúde & 1 \\
\hline & Avaliação da assistência & Organização do sistema & 1 \\
\hline 1991 & Avaliação da assistência & Abordagem antropológica & 1 \\
\hline 1992 & Atuação profissional & ACS & 1 \\
\hline \multirow[t]{2}{*}{1994} & Avaliação da assistência & Qualidade & 1 \\
\hline & Assistência à saúde & Medicina familiar e comunitária & 1 \\
\hline 1996 & Gestão & Organização do sistema de saúde & 1 \\
\hline \multirow[t]{2}{*}{1997} & Política de saúde & Saúde mental/Modelo assistencial & 2 \\
\hline & Gestão & Participação comunitária & 1 \\
\hline \multirow[t]{3}{*}{1998} & Avaliação da assistência & Satisfação de usuário/hipertensão & 2 \\
\hline & Assistência à saúde & Educação popular & 1 \\
\hline & Política de saúde & Família & 1 \\
\hline \multirow[t]{2}{*}{1999} & Assistência à saúde PSF & Violência doméstica & 1 \\
\hline & Assistência à saúde & Diabetes & 1 \\
\hline \multirow[t]{3}{*}{2000} & Política de saúde & Modelo assistencial / Modelo PSF & 2 \\
\hline & Avaliação da assistência & Materno-infantil & 2 \\
\hline & Gestão & Assistência farmacêutica & 1 \\
\hline \multirow[t]{3}{*}{2001} & Avaliação da assistência & Diabetes/ Materno-infantil & 2 \\
\hline & Assistência à saúde & Álcool/saúde mental & 2 \\
\hline & Processo de trabalho PSF & Trabalho em equipe & 1 \\
\hline \multirow[t]{7}{*}{2002} & Política de saúde & Descentralização(3)/Financiamento(2)/ & 10 \\
\hline & & $\begin{array}{l}\text { Eqüidade(1)/Modelo PSF e Medicina de Família } \\
\text { (4) }\end{array}$ & \\
\hline & Avaliação AB/PSF & Implementação da política & 2 \\
\hline & Assistência à saúde & Violência/Saúde Mental & 2 \\
\hline & Avaliação da assistência & Materno-infantil & 1 \\
\hline & Avaliação da assistência PSF & Cuidado & 1 \\
\hline & Atuação profissional PSF & ACS & 1 \\
\hline \multirow[t]{6}{*}{2003} & Politica de saúde & $\begin{array}{l}\text { Financiamento (2) /Modelo assistencial (2) / Saúde } \\
\text { do Idoso (1) }\end{array}$ & 5 \\
\hline & Assistência à saúde & $\begin{array}{l}\text { Materno-infantil (4)/ grupos populacionais } \\
\text { excluídos }\end{array}$ & 5 \\
\hline & Avaliação AB/PSF & Implementação da política & 2 \\
\hline & Assistência à saúde PSF & Saúde Mental & 1 \\
\hline & Atuação profissional & Saúde Bucal & 1 \\
\hline & Gestão & Sistema de informações & 1 \\
\hline \multirow[t]{9}{*}{2004} & Atuação profissional PSF & Ética (2) / ACS (2) / Saúde bucal / Aids & 6 \\
\hline & Processo de trabalho PSF & Cotidiano/Supervisão/Trabalho em equipe & 3 \\
\hline & Gestão & Sistemas de informações / metodologia & 2 \\
\hline & Assistência à saúde PSF & organização & 2 \\
\hline & Recursos humanos & Habitação saudável / Saúde bucal & 1 \\
\hline & Recursos humanos PSF & Formação odontólogo & 1 \\
\hline & Política de saúde & Capacitação enfermagem & 1 \\
\hline & Assistência à saúde & Financiamento & 1 \\
\hline & & Cuidado & \\
\hline 2005 & Assistência à saúde & $\begin{array}{l}\text { Saúde dos homens/materno-infantil/assistência } \\
\text { domiciliar/humanização/saúde bucal/assistência } \\
\text { farmacêutica/diabetes }\end{array}$ & 7 \\
\hline
\end{tabular}




\begin{tabular}{lll}
\hline Atuação profissional PSF & Enfermagem/nutrição/odontólogo(2) & 4 \\
Processo de trabalho PSF & Trabalho em equipe/cotidiano/processo de trabalho & 3 \\
Assistência à saúde PSF & Tuberculose/Saúde do Idoso/Cuidado & 3 \\
Avaliação da assistência & Álcool/materno-infantil & 2 \\
Avaliação da assistência PSF & Avaliação processo & 1 \\
Gestão & Sistema de informações & 1 \\
Recursos humanos & Formação do odontólogo & 1 \\
Recursos humanos PSF & Capacitação & 1 \\
\hline Avaliação AB/PSF & Implementação da política & 9 \\
Avaliação da assistência PSF & Materno-infantil/saúde do adolescente/saúde & 5 \\
& mental/dengue/diabetes & \\
Assistência à saúde & & 3 \\
Atuação profissional PSF & Saúde do idoso (2)/materno-infantil & 2 \\
Atenção primária à saúde & Saúde bucal & 2 \\
Avaliação da assistência & Materno-infantil/Saúde mental & 2 \\
Assistência à saúde PSF & Saúde mental & 1 \\
Politica de saúde & Financiamento & 1 \\
Processo de trabalho PSF & Trabalho em equipe & 1 \\
Recursos humanos & Saúde indígena & 1 \\
Recursos humanos PSF & Perfil dos profissionais & 1 \\
\hline
\end{tabular}

Fonte: Base de dados da produção dos periódicos Revista de Saúde Pública/USP, Cadernos de Saúde Pública/FIOCRUZ, Ciência e Saúde Coletiva/ABRASCO e Revista Saúde em Debate/CEBES.

A partir dos anos 2000 e principalmente a partir de 2002, há uma tendência à mudança de foco nos temas tratados, voltados para a discussão da política e estratégia de financiamento da atenção básica/PSF, avaliação da implementação da política, atuação profissional no PSF, processo de trabalho e atenção a grupos populacionais (mulher, saúde bucal, saúde mental, etc.) (quadro 1). Os temas sugerem uma inflexão dos estudos no sentido da busca de elementos para uma análise mais apurada dos desafios na implementação da política e configuração do modelo assistencial, tendo como principal elemento de análise o PSF.

Nesse contexto, há também um conjunto de artigos com discussões relativas à atuação profissional, o processo de trabalho e os recursos humanos. Estes estudos em geral apresentam discussões que buscam refletir sobre as práticas e inserção do profissional no PSF, buscando a especificidade da atuação de cada profissional; os artigos que tratam do processo de trabalho, noutra perspectiva, buscam aprofundar a organização e o modus operandi da política no âmbito da assistência e da equipe, numa aproximação com a temática do modelo assistencial a partir da perspectiva da organização do trabalho e das práticas. Essa mudança no foco dos artigos se expressa no uso das palavras-chave utilizadas nos estudos (tabela 2).

A tendência de revalorização de temas relativos à organização do cuidado na APS (da relação do profissional ou equipe de saúde com o indivíduo e sua família e organização de práticas efetivas e resolutivas na área) permeou o debate sobre 
APS nos anos 70, vinculada aos pressupostos da medicina integral e preventiva. Nos anos 80 e 90, esta discussão foi substituída por temáticas mais amplas, onde a APS passou a ser pensada numa perspectiva mais sistêmica de organização dos serviços de saúde. Com a definição da política de atenção básica e o foco no PSF, especialmente a partir dos anos 2000 , verifica-se que a discussão no sentido mais estrito da atenção à saúde volta a ser valorizada. Contudo, ainda se mostram desarticulados o discurso das práticas, cuidado e atenção do discurso da política, como se fossem áreas diferentes.

A análise dos artigos faz-nos refletir sobre o processo de reforma sanitária em curso e a necessidade de aprofundamento da discussão sobre a organização das práticas de saúde, como uma questão a ser equacionada. Nesse sentido, uma questão merece atenção: boa parte dos estudos realizados no final dos anos 90 e na década de 2000 acompanha e reforça as diretrizes da política traçada pelo Ministério da Saúde. Os textos dos anos 2000 apresentam como principal característica o discurso da política oficial, onde se assume como dado o que diz a política. Em muitos artigos, o referencial teórico é uma síntese da política nacional do PSF, dos seus princípios e diretrizes, estando a discussão da APS

\section{Tabela 2 - Produção bibliográfica em APS no Brasil em quatro periódicos nacionais conforme palavras-chave utilizadas e ano da publicação}

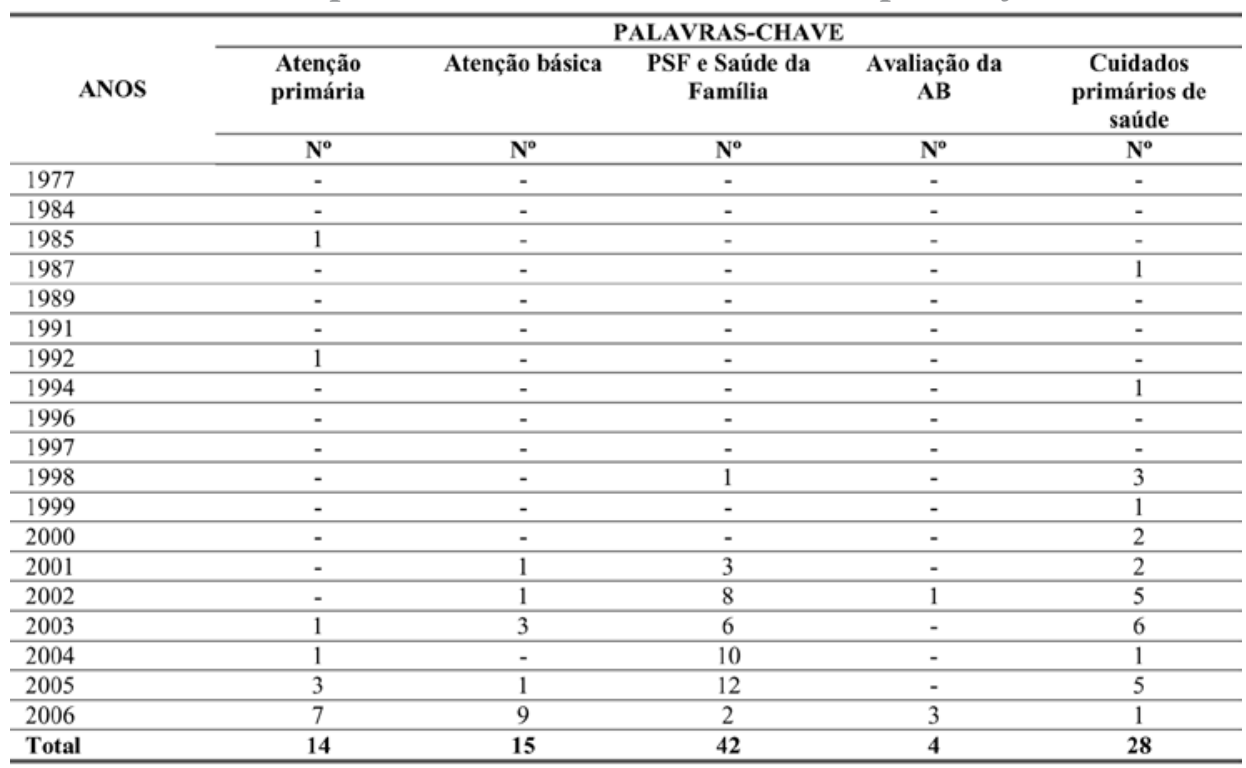

Fonte: Base de dados da produção dos periódicos Revista de Saúde Pública/USP, Cadernos de Saúde Pública/FIOCRUZ, Ciência e Saúde Coletiva/ABRASCO e Revista Saúde em Debate/CEBES. 
subentendida nesse debate. Mesmo estudos que se propõem a avaliar aspectos da

APS não apresentam uma base conceitual que informe a compreensão adotada, bem como a abrangência conferida a APS. Este é um aspecto importante, uma vez que há distintas maneiras de se conceber APS.

\section{Conceito e atributos da APS}

Outra forma de olhar os artigos é a busca do conceito, atributos e características da APS utilizados pelos autores. Dois terços dos estudos (68,2\%) apresentaram discussōes conceituais relativas à APS (tabela 3). No conjunto, apenas dois artigos (GIL, 2006; FERNANDES, 1992) apresentaram como proposta uma reflexão acerca desta área e somente um artigo se dedicou a pensar a APS no âmbito internacional (GIOVANELLA, 2006). Na análise sobre os conceitos e atributos da APS, foi possível extrair algumas especificidades da discussão brasileira.

Um primeiro aspecto a destacar é a relação da APS com o princípio da integralidade, referida em 42,6\% dos artigos (tabela3). Esse é um aspecto importante da produção de APS no Brasil desde os anos 70 e constitui um diferencial em relação aos demais países, pois se fala em extensão de cobertura com a garantia de um leque abrangente de açôes e serviços de saúde, com o repúdio à definição de mínimos. Foram raros os artigos que ousaram propor a focalização ou a cesta

\section{Tabela 3 - Conceitos e atributos da APS na produção bibliográfica de quatro periódicos nacionais - Brasil - (1977-2006)}

\begin{tabular}{lc}
\hline \multicolumn{1}{c}{ Conceito e atributos da APS } & $\%$ artigos \\
\hline A APS visa a integralidade da atenção & $42,6 \%$ \\
A APS tem uma relação direta com comunidade/território & $31 \%$ \\
A APS é uma estratégia de reorientação do modelo a partir do PSF/PACS & $29,5 \%$ \\
A APS visa ampliar a cobertura assistencial & $24,3 \%$ \\
A APS é uma estratégia de reorientação do modelo tendo como principal eixo a prevenção e & $22,6 \%$ \\
promoção da saúde & $21,7 \%$ \\
A APS corresponde ao primeiro nível da atenção & $17,8 \%$ \\
A APS é a porta de entrada para o sistema de saúde & $13,2 \%$ \\
A APS visa reduzir a desigualdade de acesso & $11,6 \%$ \\
A APS é uma estratégia para a organização da rede & $1,6 \%$ \\
A APS refere-se a um conjunto de mínimo de ações, focais & $31,8 \%$ \\
O artigo não abordou conceito e atributos da APS & $2 \%$ \\
\hline
\end{tabular}

Fonte: Base de dados da produção dos periódicos Revista de Saúde Pública/USP, Cadernos de Saúde Pública/FIOCRUZ, Ciência e Saúde Coletiva/ABRASCO e Revista Saúde em Debate/CEBES. 
básica como uma característica da APS (1,6\%), estando presente especialmente em análises da política e particularmente do financiamento nos anos 2000.

A temática da integralidade também está presente nos artigos que ressaltam como objetivo da APS ampliar a cobertura assistencial (24,3\%) e reduzir as desigualdades de acesso aos serviços (13,2\%). No Brasil, estas são questōes importantes para o pensamento sobre APS desde os anos 70 e ganham maior expressão nos anos 90 , quando a equidade passa a ser um princípio fundamental para reforma do sistema de saúde nacional.

Outro tópico importante que caracteriza a produção de APS no Brasil é a relação com a noção de território presente em $31 \%$ dos artigos. A aproximação com a realidade social, cultural e ambiental do indivíduo, sua família e a comunidade tem sua origem na medicina integral de onde se constituiu o arcabouço teórico-conceitual da APS (AROUCA, 2003). Nos anos 80, a ideia de definição de territórios esteve fortemente presente nas propostas de construção dos Sistemas Locais de Saúde e de sua organização a partir da estruturação dos Distritos Sanitários (MENDES, 1994). Esta proposta pressupunha a definição de clientela, formando forte vínculo com a comunidade e construindo o que se convencionou chamar de responsabilidade sanitária. Essa é uma discussão atual e permeia o debate sobre a descentralização da saúde e organização da assistência, na relação com a comunidade e na definição de estratégias para a regionalização da saúde.

Um terceiro aspecto a ressaltar na produção é a compreensão da APS como uma estratégia para reorientação do modelo a partir do PACS/PSF, entendendo que estes programas constituem a melhor forma para a organização da APS no Brasil. APS e PACS/PSF são tratados como sinônimos na produção, o que está expresso em 29,5\% dos artigos. O principal marco teórico desses artigos é a política ministerial. Considerando que há uma trajetória de organização da APS no Brasil e que o PACS/PSF não é necessariamente a única forma de organização da atenção, é preciso que se aprofunde o debate.

A perspectiva de reorientação do modelo a partir da APS também está expressa em textos $(22,6 \%)$ que abordam a APS como uma estratégia de reorientação que tem como principal eixo a prevenção e promoção da saúde. Nesse sentido, nos anos 2000 a APS assume papel de destaque na organização do sistema de saúde, tendo como principal missão a reorientação do modelo assistencial. 
Seguindo a perspectiva de Vuori (1986), nos artigos revisados houve o predomínio da discussão sobre a organização da atenção, como apontado acima, mas também da noção de APS como primeiro nível de atenção $(21,7 \%)$ e porta de entrada (17,8\%) do sistema de saúde (tabela 3).

É interessante destacar, na produção, a ênfase dada à organização do sistema e a ausência de debate acerca das ações a serem empreendidas. Parece haver uma lacuna de discussão sobre as práticas e a organização da assistência propriamente dita. Nos artigos que abordam a assistência, o que prevalece é a perspectiva eminentemente clínica sem uma contribuição teórica mais específica sobre a organização da atenção na perspectiva da APS. Configuram-se dois eixos de discussão que não necessariamente dialogam entre si: os estudos que tratam da clínica no âmbito da APS e os estudos que abordam a política e o modelo assistencial na perspectiva macro do sistema. Além disso, há uma carência de diálogo dos estudos nacionais com as experiências internacionais restringindo o debate à perspectiva do sistema nacional.

Nos anos 2000, há uma inflexão da produção na tentativa de aprofundar o modelo assistencial para além da perspectiva da macropolítica, buscando elementos para avaliação da APS. Neste cenário, torna-se referência importante a produção de Starfield (2002), divulgada pelo Ministério da Saúde em parceria com a UNESCO. A autora tem contribuído principalmente na formulação da base conceitual e metodológica para os estudos avaliativos em APS no mundo. Uma contribuição importante no Brasil num momento que se exigiam respostas de avaliação da política.

A leitura de Starfield (2002) significou uma retomada do debate conceitual acerca do modelo assistencial e da APS, resgatando aspectos da assistência propriamente dita: a atenção centrada na família e orientada para a comunidade, a atenção de primeiro contato, o vínculo, a integralidade e a continuidade do cuidado. Nota-se que parte dos aspectos que caracterizam a APS colocados por Starfield está presente nos estudos revisados desde os anos 70, ainda que não colocados de maneira sistematizada, mas somente os estudos elaborados na última década (2000) utilizam a autora como referência e exploram conceitualmente tais características. 


\section{Autorias, rede de conhecimento e abrangência dos estudos}

Quanto à identificação da autoria e construção da rede de conhecimento, o que se verificou nestes veículos foi a existência de uma pulverização de autores e instituições que abordam a APS no Brasil, especialmente a partir de 2002, quando a produção se intensifica. Não há um núcleo duro de pesquisa com pesquisadores líderes nessa área, mas trabalhos que se desenvolvem no âmbito das diversas instituições, com autorias diversas. A pulverização da produção por instituições e autores fragiliza o debate conceitual e favorece a dispersão do conhecimento, o que também gera um diálogo pequeno entre grupos. Há que se considerar que esta análise se restringe a quatro periódicos nacionais e que há um conjunto expressivo de trabalhos publicados em livros, além da produção de dissertação e teses, sob a orientação de muitos autores desses artigos.

Por outro lado, há uma distribuição mais balanceada da produção pelo território nacional, antes concentrado no Sudeste e nas grandes instituições (tabela 4), o que é positivo para a ampliação da rede de conhecimento e produção em APS. Percebe-se, por exemplo, um maior número de instituições do Sul (principalmente) e Nordeste na produção, bem como a participação de faculdades particulares (6,3\% dos artigos), secretarias de Saúde (3,5\%) e associações de moradores $(2,1 \%)$, num processo de democratização da produção, o que poderá

\begin{tabular}{|c|c|c|c|}
\hline Regiões & Principais instituições & $\mathbf{N}^{\circ}$ & $\%$ \\
\hline Norte & UFPa Univ.Estadual da Zona Oeste e Cpqam/Manaus & 2 & 1,4 \\
\hline Nordeste & UFPi UFAl UFPb UFBa UFRN UFPE & 22 & 15,4 \\
\hline Centro-Oeste & Unb, SES-DF & 3 & 2,1 \\
\hline Sudeste & USP ENSP FIOCRUZ UFF UNICAMP FESO UFRJ & 80 & 55,9 \\
\hline Sul & UFPel UEL UFRS UFSC & 30 & 21,0 \\
\hline Internacional & Harvard School Un de LiègeBelgica; Johns Hopkins & 3 & 2,1 \\
\hline Nacional & MS ANS & 3 & 2,1 \\
\hline Total & & 143 & 100 \\
\hline
\end{tabular}

Fonte: Base de dados da produção dos periódicos Revista de Saúde Pública/USP, Cadernos de Saúde Pública/FIOCRUZ, Ciência e Saúde Coletiva/ABRASCO e Revista Saúde em Debate/CEBES.

Nota: $\mathrm{O}$ número total corresponde à participação das instituições na autoria dos artigos e não ao número total de artigos revisados. 
fortalecer a constituição de núcleos de pesquisa nessa área. Contudo, poucos artigos foram produzidos em parceria com Instituições internacionais (2,1\%), o que também denota uma produção muito endógena.

Outra característica importante da produção refere-se à abordagem dos estudos. Há uma abundância de estudos de caso (74,4\% dos artigos) com uma diversificação de objetos empíricos. A produção se expande de forma horizontal, o que se expressa num conjunto de estudos sobre o mesmo tema e que dialogam pouco entre si, trazendo reflexos para a produção no sentido do aprofundamento de conceitos e marcos teóricos. Essa é uma tendência não só da produção desta área temática, mas que já foi apontada em estudo anterior sobre a produção em política, planejamento e gestão em Saúde Coletiva (LEVCOVITZ et al., 2003), como também na análise da produção em políticas públicas no Brasil (SOUZA, 2003). Nesse sentido, observa-se que a crescente produção sobre APS precisa avançar para uma discussão de questóes conceituais, bem como promover o diálogo entre experiências nacionais e internacionais.

\section{Considerações finais}

São dois grandes momentos da discussão sobre atenção primária no Brasil. Um primeiro, localizado nos anos 1970, com características que se assemelhavam à agenda internacional, onde a questão em foco era o fortalecimento das ações de promoção e prevenção em saúde e a extensão da cobertura. Um segundo momento, a partir da segunda metade dos anos 1990, quando o PSF assume centralidade na agenda governamental e se define como estratégia de reorganização da atenção básica no âmbito do SUS. A concepção de APS é vista como nível de atenção e estratégia de reorientação do sistema de saúde e estas ações devem ser dirigidas a toda população, havendo, entre elas, aquelas ações dirigidas aos grupos específicos.

O que se depreende deste estudo é que essa é uma área em formação, com escassa acumulação de conhecimento e que merece um esforço de construção teórica. É necessário o fortalecimento deste campo como um programa de pesquisa (LAKATOS, 1979), com maior envolvimento da comunidade de pesquisadores, diálogo e troca de conhecimento. O esforço de consolidação dessa área é muito recente, e por estar atrelado a uma política governamental, tem sofrido as consequências dessa proximidade, sendo pouco crítico à realidade e defensor da política oficial. A proximidade de uma área de pesquisa com os órgãos governamentais pode tanto gerar trabalhos normativos e prescritivos, 
como abrir a possibilidade de esses órgãos estimularem a agenda de pesquisa. No entanto, é preciso um esforço próprio do campo para a construção do referencial teórico, de modo a possibilitar o diálogo com os diversos grupos e interesses (internos e externos ao campo) sem necessariamente estar subjugado ao poder político. A especificidade da Saúde Coletiva no Brasil é também uma questão a ser considerada, por ser este um campo de pesquisa e ação, com forte vocação política (PAIM; ALMEIDA FILHO, 2000).

No entanto, a carência de estudos na área não é uma prerrogativa brasileira. Uma revisão sistemática da literatura sobre APS em países europeus e nos Estados Unidos realizada por Atun (2004) revelou a escassez de estudos qualificados que apontem as vantagens e desvantagens dos modelos de atenção baseados em APS, comparativamente aos pautados no cuidado dos especialistas, embora o autor observe um aumento na complexidade e qualidade dos estudos no período analisado. Também Harzheim et al. (2005) apontam que a investigação na área de APS no Brasil carece de investimentos maiores e de uso de instrumental metodológico que fomentem análises mais contributivas para a qualificação do processo de decisão que regulamenta a política setorial.

A análise da produção aqui empreendida mostra uma lacuna da discussão sobre os conceitos e formas de estruturação da APS no Brasil, cujo enfoque está no PSF e na reorientação/conversão do modelo, sem especificar a discussão que embasa o modelo. Há uma tendência em achar esta uma discussão menor, fruto talvez de um repúdio da discussão sobre APS recorrente nos anos 80 , onde a atuação do Estado na APS tinha características seletivas, restrita a um pacote de serviços, conforme as recomendaçôes de agências internacionais (FAUSTO, 2005). No entanto, não há duvidas de que o PSF teve papel fundamental no resgate da APS no Brasil, não somente na agenda da política nacional, mas também na agenda da produção acadêmica, sugerindo ser este um campo de investigação bastante fértil e que merece um olhar mais acurado, principalmente para as lacunas de investigação identificadas.

Apesar da importância desses estudos, uma vez que a política ainda está em fase de implementação, faz-se necessário investir em pesquisas que apontem para a qualificação da APS no sistema de saúde brasileiro, na capacidade resolutiva do modelo centrado na APS, já que este é o principal argumento para o direcionamento dos investimentos recentes feitos pelo Estado no setor de saúde. 


\section{Referências}

AROUCA, A.S.S. O dilema preventivista. Contribuição para a compreensão da medicina preventiva. Rio de Janeiro: Fiocruz, 2003. 268p.

ATUN, R. What are the advantages and disadvantages of restructuring a health care system to be more focused on primary care services? WHO Regional Office for Europe's Health Evidence Network (HEN), 2004. Disponível em: <http:/www.euro.who.int/HEN/Syntheses/ primaryvsspecialist/20040115_15>. Acesso em: 04 nov. 2004.

BANCO MUNDIAL. Informe sobre o Desenvolvimento Mundial 1993: Investindo em Saúde. Washington: Banco Mundial; 1993. 344p.

CANESQUI, A.M.; OLIVEIRA, A.M.F. Saúde da família: modelos internacionais e estratégia brasileira. In: NEGRI, B.; VIANA, A.L.D. (Org.). O Sistema Único de Saúde em dez anos de desafios. São Paulo: Sobravime, 2002, p. 241-270.

CONILL, E.M.; FAUSTO, M.C.R. Analisis de la problemática de la integración APS en el contexto actual: causas que inciden en la fragmentación de servicios y sus efectos en la cohesión social. Rio de Janeiro: Intercâmbio EuroSocial Salud, 2007.68p.

CUETO, M. The origins of primary health care and selective primary health care. Lima: Universidad Peruana Cayetano Heredia; 2003. Disponível em: http://www.globalhealthtrust. org/doc/Word\%20Marcos\%20Cueto.pdf. Acesso em: 21 ago 2004.

FAUSTO, M.C.R. Dos programas de medicina comunitária ao Sistema Único de Saúde: uma análise histórica da atenção primária na política de saúde brasileira. 2005. 261p. Tese (Doutorado em Saúde Coletiva) - Instituto de Medicina Social, Universidade do Estado do Rio de Janeiro, Rio de Janeiro, 2005.

FERNANDES, J.C.L Agentes de saúde em comunidades urbanas. Cadernos de Saúde Pública, Rio de Janeiro, v. 8, n. 2, p. 134-139, 1992.

FUNDO DAS NAÇÕES UNIDAS PARA A INFÂNCIA. Cuidados Primários de Saúde. Relatório da Conferência Internacional sobre Cuidados Primários de Saúde - Alma-Ata, 1978. Brasília: Unicef; 1979. 64p.

GIL, C.R.R. Atenção primária, atenção básica e saúde da família: sinergias e singularidades do contexto brasileiro. Cadernos de Saúde Pública, v. 22, n. 6, p. 1.171-1.181, 2006.

GIOVANELLA, L. A atenção primária à saúde nos países da União Europeia: configurações e reformas organizacionais na década de 1990. Cadernos de Saúde Pública, v. 22, n. 5 , p. 951-963, 2006.

HARZHEIM, E et al. Revisão sistemáticas sobre aspectos metodológicos das pesquisas em atenção primária no Brasil. Revista AMRIGS, Porto Alegre, v. 49, n. 4, p. 248, 2005.

KINGDOM, J.W. Agendas, alternatives and public policies. New York: Harper Collins, 1995.

LAKATOS, I. O falseamento e a metodologia dos programas de pesquisa científica. In: 
LAKATOS, I.; MUSGRAVE, A. (Org.). A crítica e o desenvolvimento do conhecimento. São Paulo: Cultrix, 1979. p. 109-244.

LEVCOVITZ, E et al. Produção de conhecimento em politica, planejamento e gestão em saúde e politicas de saúde no Brasil: 1974-2000. Brasília: OPAS, 2003 (Série Técnica - Projeto de Desenvolvimento de Sistemas e Serviços de Saúde, n. 2).

LEVCOVITZ, E.; GARRIDO, N. G. Saúde da Família: à procura de um modelo anunciado. Cadernos de Saúde da Família. V. 1. Brasília: Ministério da Saúde; 1996.

MAGNUSSEN, L.E.J.; JOLLY, P. Comprehensive versus selective primary health care: lessons for global health policy. Health Affairs, v. 23, n. 3, p. 167-176, 2004.

MENDES, E.V. (Org.). Distrito Sanitário: o processo social de mudança das práticas sanitárias do Sistema Único de Saúde. São Paulo: Hucitec, 1994. 310p.

NOGUEIRA, D.P. Incorporação da saúde ocupacional à rede primária de saúde. Revista de Saúde Pública, v. 18, n. 6, p. 495-509, 1984.

NORONHA, J.C. et al. Transformações de um ambulatório de medicina integral com vistas a um programa de atenção médica primária. Revista de Saúde Pública, v. 11, p. 429-443, 1977.

ORGANIZAÇÃO MUNDIAL DA SAÚDE. Atenção primária de saúde nas Américas: as lições aprendidas ao longo de 25 anos e os desafios futuros. Washington: WHO, 2003. Disponível em: http://www.paho.org/portuguese/gov/cd/cd44-r6-p.pdf. Acesso em: 28 out 2004 .

PAIM, J.; ALMEIDA FILHO, N. A crise da saúde pública e a utopia da saúde coletiva. Salvador: Casa da Qualidade, 2000. 123p.

PAN AMERICAN HEALTH ORGANIZATION. Health in the Americas: 2007. Washington DC: PAHO, 2007. (Paho Scientific and Technical Publication n. 622). 747p.

PAN AMERICAN HEALTH ORGANIZATION. Primary Health Care in the Americas: conceptual framework, experiences, challenges and perspectives. Washington DC: PAHO; 2002. 580p.

SOUZA, C. "Estado do campo" da pesquisa em políticas públicas no Brasil. Revista Brasileira de Ciências Sociais, v. 18, n. 51, p. 15-20, 2003.

SOUZA, H.M. Saúde da família: desafios e conquistas. In: NEGRI, B.; VIANA, A.L.D. (Org.). O Sistema Único de Saúde em dez anos de desafios. São Paulo: Sobravime, 2002. p. 221-240.

STARFIELD, B. Atenção primária: equilíbrio entre necessidades de saúde, serviços e tecnologia. Brasília: Ministério da Saúde, 2002. 725 p.

VUORI, H. Health for all, primary health care and general practitioners. Journal of the Royal College of General Practitioners. London, n. 36, p. 398-402, 1986. 
WORLD HEALTH ORGANIZATION. World Health Report 2000. Health systems: Improving performance. Geneva, 2000. Disponível em: http://www.who.int/whr2001/2001/ archives/2000/en/index.htm. Acesso em: 09 set 2004.

OMS-UNICEF, 1979. Declaração de Alma-Ata. Conferência Internacional sobre Cuidados Primários da Saúde, Alma-Ata, URSS, 6 a 12 de setembro de 1978. 
Review of scientific literature on primary health care in Brazil in four selected journals This paper analyzes the scientific production in primary care in Brazil published in four magazines with wide circulation in the field of Public Health - Cadernos de Saúde Pública, Revista de Saúde Pública, Ciência \& Saúde Coletiva and Saúde em Debate - in order to identify the concepts used, the approaches to studies, and the main issues associated with the discussion of primary care. The analysis was based on the understanding of the political and institutional context of health, an attempt to correlate the time of policy with knowledge production. The analysis covered the discussions held in these journals from 1977 to 2006. There was a lack of studies of conceptual approach and many case studies without the promotion of dialogue between authors and institutions, weakening this area as a knowledge producer. This study aims to contribute to a deeper debate on the theoretical model of care focused on primary care and to identify the strength of the proposals, thus contributing to consolidate the field.

> Key words: primary health care; family health care; knowledge production. 